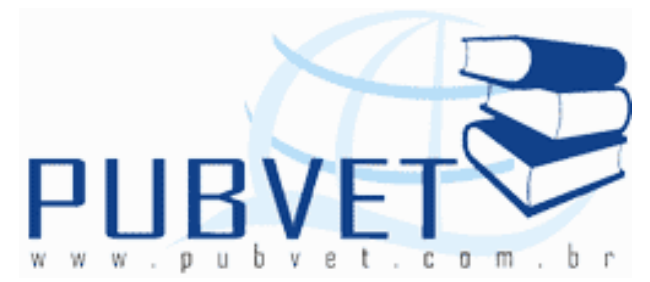

PUBVET, Publicações em Medicina Veterinária e Zootecnia.

\title{
Anatomia vascular de Podocnemis unifilis (Testudines - Pelomedusidae). Comportamento da artéria aorta.
}

\author{
André Luiz Quagliatto Santos ${ }^{1}$, Lorena Tannús Menezes ${ }^{2}$, Priscilla Inocêncio \\ Rodrigues Ribeiro ${ }^{2}$, Liliane Rangel Nascimento², Arthur Paulino Sanzo \\ Kaminishi ${ }^{2}$, Tatiana Grillo Leonardo ${ }^{2}$.
}

Laboratório de Ensino e Pesquisas em Animais Silvestres - LAPAS, FAMEV/UFU, e-mail: quagliatto@famev.ufu.br 1. Docente. 2. Mestrandos.

\section{Resumo}

Este trabalho estudou o comportamento da artéria aorta, focalizando sua origem, trajeto, e destino de suas ramificações. Utilizou-se cinco exemplares de Podocnemis unifilis, fêmeas, adultas, doadas pelo CENAQUA (Centro Nacional de Quelônios da Amazônia) situado na cidade de Goiânia-GO-Brasil. Canulou-se as artérias carótidas comuns através das quais se introduziu solução fisiológica a fim de desobstruir o sistema vascular, em seguida foi aplicado solução de Neoprene Látex "450" corada. Fixou-se o material preparado em solução de Formol a 10\% por um período de 96 horas, após este desprendeu-se a carapaça de suas inserções, evidenciando as vísceras, e individualizamos a artéria aorta. Observou-se que os arcos aórticos direito e esquerdo unem-se em um ponto após sua origem no coração. O arco aórtico esquerdo emite a artéria gástrica, que surge cranialmente ao ponto de união dos arcos aórticos. Emite a artéria celíaca, que fornece as artérias 
SANTOS, A.L.Q. et al. Anatomia vascular de Podocnemis unifilis (Testudines - Pelomedusidae). Comportamento da artéria aorta. PUBVET, Londrina, V. 5, N. 16, Ed. 163, Art. 1103, 2011.

pancreaticoduodenais cranial e caudal e as artérias mesentéricas cranial e caudal. Após a união dos dois arcos aórticos forma-se a artéria aorta e originam-se desta as artérias renais, epigástricas, ilíacas comuns e a artéria caudal. O arco aórtico direito não emite nenhum ramo antes da sua união com o arco aórtico esquerdo, portanto os principais ramos viscerais surgem do arco aórtico esquerdo ou diretamente da artéria aorta.

Palavras-chave: anatomia, Podocnemis unifilis, artéria aorta, repteis.

\title{
Vascular anatomy of the Podocnemis unifilis (Testudines- Pelomedusidae). Aorta artery behaviour
}

\begin{abstract}
This research studied the behaviour of the artery aorta, focing in the origin, trajectory, and destiny of the ramifications. Was used five turtles Podocnemis unifilis, females, adults, donated by CENAQUA (National Center of Amazon Quelonians) situated in the city of Goiania-GO-Brazil. The common carotid arteries were being canulate and a physiological solution was introduced to remove obstruction of the vascular system, than was injected solution of Neoprene Latex "450" colored. The material prepared was fixed in solution of Formaldehyde $10 \%$ for a period of 96 hours. After this, was loosed the skull from their insertions, showing the viscera, and individualizing the aorta artery. The right and left aortic arch unite in a single point after their origin in the heart. The left aortic arch emits the gastric artery that arise cranially to the point of union of the aortic arcs. It still emits a celiac artery that gives the cranial and caudal pancreaticduodenal arteries and the cranial and caudal mesenteric arteries. After the union of the two aortic arch was formed the aorta artery and from it originated the renal arteries, epigastrics, common iliacs and the caudal artery. The aortic right arch does not emit any branch before its union with the left aortic arch, so the main visceral branches come from the left aortic arch or directly from the aorta artery.
\end{abstract}

Keywords: anatomy, Podocnemis unifilis, aorta artery, reptiles. 


\section{INTRODUÇÃO}

Os répteis provocam todos os tipos de reações nas pessoas, uma vez que são grupos de animais pouco compreendidos e escassamente conhecidos.

Nos dias de hoje podemos considerar que os répteis são pouco numerosos, pois se comparando ao período cretáceo, por exemplo, denominado de "A idade dos répteis", de 135 a 70.000.000 anos atrás, eram os animais dominantes na Terra (BURTON e BURTON, 1984; CARR, 1981; FUENTE, 1982; ORR, 1986; PRITCHARD, 1993).

Após esta era, a maioria sucumbiu, mas os que conseguiram sobreviver evoluíram em dura competição com aves e mamíferos, modificando suas estruturas e alterando sua fisiologia até conseguir uma adaptação em quase todos os biótipos terrestres e aquáticos do mundo contemporâneo (FUENTE, 1982). Porém é uma classe pouco estudada se comparada a peixes, aves e mamíferos.

A espécie a qual propomos à estudar, Podocnemis unifilis, é um quelônio da fauna brasileira pertencente ao filo Chordata, ao subfilo Vertebrata, a superclasse Tetrapoda, a classe Reptila e a ordem Chelonia (ASHEY, 1969; AMABIS e MARTHO, 1990; BENZZONI, 1972; CUNHA, 1969; HICKMAN, s.d; MORANDINI, 1968; GRASSÉ, 1978; ORR, 1986; POUGH et al., 1993; RENNER e ROMER, 1973; ROMER e PARSONS, 1985; STORER et al., 1995; ZISWILLER, 1978).

Como características gerais, a Podocnemis unifilis apresenta um corpo inteiramente coberto por um casco, também denominado carapaça superior ou casco e o plastrão na porção inferior, que são fortes de onde somente a cabeça, os membros e a cauda emergem. A carapaça e o plastrão são formados por placas ósseas poligonais, soldadas.

De acordo com Breland (1953), o arco sistêmico origina as artérias gástricas, celíaca e mesentérica superior, onde a artéria gástrica supre o estômago, a celíaca o duodeno, pâncreas, parte posterior do estômago e estruturas associadas, e a mesentérica superior o intestino. O autor, ainda, 
SANTOS, A.L.Q. et al. Anatomia vascular de Podocnemis unifilis (Testudines - Pelomedusidae). Comportamento da artéria aorta. PUBVET, Londrina, V. 5, N. 16, Ed. 163, Art. 1103, 2011.

relata que a aorta dorsal origina a artéria ilíaca interna que irriga o intestino grosso.

Harrison (1964) comenta que a aorta dorsal dá origem á artéria celíaca, a qual origina o ramo gástrico anterior, o ramo pancreáticoduodenal e o ramo hepático para seus respectivos órgãos; e a artéria mesentérica anterior para o fígado e intestino delgado.

Baroudi (1965) afirma que as duas aortas depois de formarem a respectiva crossa, unem-se formando a aorta torácica única, que desce pelo tronco emitindo ramos em todo o seu trajeto e irrigando as estruturas do organismo. Nielsen (1986), Storer et al. (1995) relatam que os dois arcos aórticos unem-se numa aorta dorsal que distribui sangue para os órgãos da cavidade do corpo, para os membros posteriores e para a cauda. Entretanto Neal e Rand (1954), Hyman (1957) comentam que o arco aórtico esquerdo origina as artérias celíaca e mesentérica anterior, as quais carreiam sangue até o estômago e intestino e que os arcos aórticos direito e esquerdo originam-se do ventrículo direito e esquerdo respectivamente, unindo-se em um tronco comum próximo da parte caudal do estômago.

Faria (2000) cita que a partir da artéria celíaca tem-se origem as artérias mesentérica inferior e superior.

Levando-se em consideração a importância do conhecimento do sistema circulatório em especial o comportamento da artéria aorta destes animais, que funciona como um meio de transporte do sangue rico em nutrientes (sais minerais, carboidratos, aminoácidos, vitaminas, proteínas, etc.), excretas, hormônios e componentes do sistema imunológico, é que os pesquisadores do Núcleo de Pesquisa em Animais Silvestres da Faculdade de Medicina Veterinária da Universidade Federal de Uberlândia visam com a presente pesquisa descrever a, origem e distribuição dos principais ramos da artéria aorta na cavidade celomática, bem como enriquecer a literatura sobre a anatomia de répteis. 


\section{MATERIAL E MÉTODOS}

Utilizou-se cinco exemplares de Podocnemis unifilis (Tracajá), fêmeas e adultas. Estes quelônios foram doados pelo CENAQUA (Centro Nacional de Quelônios da Amazônia) situado na cidade de Goiânia-GO.

Identificou-se estes animais e encaminhou-se ao Laboratório de Pesquisa em Animais Silvestres da Faculdade de Medicina Veterinária da Universidade Federal de Uberlândia, onde dissecou-se e canalizou-se as artérias carótidas comuns com cânulas de polietileno de $2 \mathrm{~mm}$ de diâmetro por $50 \mathrm{~mm}$ de comprimento, através das quais introduziu-se solução fisiológica a fim de desobstruir (lavar) o sistema vascular, em seguida aplicou-se solução de Neoprene Látex "450"(Du Pont do Brasil S.A- Indústrias Químicas) coradas com pigmento específico (Globo S/A Tintas e Pigmentos).

Fixou-se o material assim preparado em solução de formol a $10 \%$ por um período mínimo de 96 horas. Após completado o tempo de fixação retirou-se o plastrão do animal, desprendendo-o da carapaça e das suas inserções na região cervical, nos membros e na cauda. Feito isto, as vísceras ficaram expostas e presas apenas na carapaça, onde se observou a artéria aorta bem como seus principais ramos. A partir deste momento procedeu-se a dissecação com pinças, bisturi e tesouras cirúrgicas, utilizou-se lupa Wild (10X) para melhor visualização da artéria aorta, focalizou-se a sua origem até sua terminação registrando-se suas relações, e o comportamento de cada um, dos seus ramos.

\section{RESULTADOS}

Os resultados se basearam em observações macroscópicas realizadas pela vista dorsal dos animais.

Analisando as peças dissecadas, pode-se verificar que os arcos aórticos direito e esquerdo unem-se em um ponto, logo após deixarem o ventrículo cardíaco direito e esquerdo, obedecendo ao trajeto dorso-cranial e ventro- 
caudal nas mediações da parte cranial do estômago. Próximo ao ponto de união dos dois arcos aórticos (na parte caudal do estômago) e cranialmente a este, o arco aórtico esquerdo emite ramos para a irrigação e para algumas vísceras, enquanto que o arco aórtico direito não emite nenhum ramo antes da sua união com o arco aórtico esquerdo. Após a união dos arcos aórticos ocorre a formação da artéria aorta de onde se originam ramos para a região caudal do corpo do animal.

Para melhor descrever os trajetos destes vasos, optou-se por fazê-lo separadamente desde sua origem no coração.

\section{ARTÉRIA GÁSTRICA}

A artéria gástrica surge cranialmente ao ponto de união dos dois arcos aórticos, emergindo do arco aórtico esquerdo.

\section{ARTÉRIA CELÍACA}

A artéria celíaca originou-se a partir do arco aórtico esquerdo em sua porção terminal, tendo aquela um menor calibre, seguindo na direção caudolateral esquerda, passando ventralmente a artéria aorta, iniciando a emissão de vários ramos a partir da região mediana, dos quais identificou-se:

\section{ARTÉRIA PANCREÁTICODUODENAL CRANIAL:}

É o primeiro ramo a emergir da face lateral esquerda da artéria celíaca, seguindo em direção crânio - lateral esquerda, sendo envolvida pelo baço e envia a este, ramos esplênicos continuando seu trajeto ao estômago.

\section{ARTÉRIA PANCREÁTICODUODENALCAUDAL:}

Parte da face lateral esquerda da artéria celíaca, em direção caudo-lateral direita, enviando um ramo para o pâncreas e outro para o duodeno. 
ARTÉRIA MESENTÊRICA CRANIAL:

Emerge em direção caudo-lateral esquerda, sendo responsável pela nutrição do mesentério, intestino delgado e parte do intestino grosso.

\section{ARTÉRIA MESENTÉRICA CAUDAL:}

Apresentou-se como continuação da artéria celíaca, sendo responsável pelo abastecimento sanguíneo de parte do intestino grosso e mesentério.

\section{ARTÉRIA AORTA}

Denomina-se de artéria aorta, a artéria que se inicia à partir da junção dos arcos aórticos esquerdo e direito e representam um importante vaso que supre de sangue a região médio-caudal do corpo do animal e dela partem artérias para as vísceras, membros pelvinos, gordura lateral, músculos longitudinais, casco e cauda.

\section{ARTÉRIAS RENAIS}

Partindo da face ventral da artéria aorta, em direção aos rins, direito e esquerdo, observaram-se artérias renais direita e esquerda em número de duas para cada rim.

Para ambos os rins encontraram-se, também, a partir das artérias ilíacas comuns esquerda e direita, um ramo renal para cada órgão.

\section{ARTÉRIAS GONODAIS}

Chamadas de ovarianas nas fêmeas ou testiculares nos machos, as artérias gonadais surgiram à partir das artérias renais, próximas ao hilo renal, sendo uma para cada gônada.

\section{ARTÉRIAS EPIGÁSTRICAS}

Acompanhando-se o trajeto da artéria aorta, logo após as artérias renais, encontrou-se a origem das artérias epigástricas esquerda e direita. Estas seguiam em direção lateral esquerda e direita respectivamente e se 
SANTOS, A.L.Q. et al. Anatomia vascular de Podocnemis unifilis (Testudines - Pelomedusidae). Comportamento da artéria aorta. PUBVET, Londrina, V. 5, N. 16, Ed. 163, Art. 1103, 2011.

bifurcavam em artérias marginocostal cranial e marginocostal caudal para seguir em direção cranio-lateral e caudo-lateral respectivamente, irrigando as margens do casco e músculos pélvicos.

\section{ARTÉRIAS ILÍACAS COMUNS}

A artéria aorta, em sua porção terminal emite as artérias ilíacas comuns esquerda e direita.

As artérias ilíacas comuns esquerda e direita constituem troncos comuns e emitem à partir de suas faces laterais direita e esquerda um ramo renal direito e um ramo lateral esquerdo, antes de formar as artérias ilíacas internas e externas.

As artérias ilíacas internas direita e esquerda se destinavam para, parte dos genitais e vesícula urinária.

As artérias ilíacas externas direita e esquerda apresentam-se como continuação das artérias ilíacas comuns direita e esquerda. As ilíacas externas direita e esquerda emitiram dois ramos, sendo as artérias femoral e isquiádica.

\section{ARTÉRIA CAUDAL}

A artéria caudal é um fino segmento da porção final da artéria aorta, que surge logo após esta e emite as artérias ilíacas comuns. 
SANTOS, A.L.Q. et al. Anatomia vascular de Podocnemis unifilis (Testudines - Pelomedusidae). Comportamento da artéria aorta. PUBVET, Londrina, V. 5, N. 16, Ed. 163, Art. 1103, 2011.

As figuras abaixo ilustram o sistema arterial da Podocnemis unifilis, focalizando os arcos aórticos direito e esquerdo, artéria gástrica e ilíacas comuns, sendo:

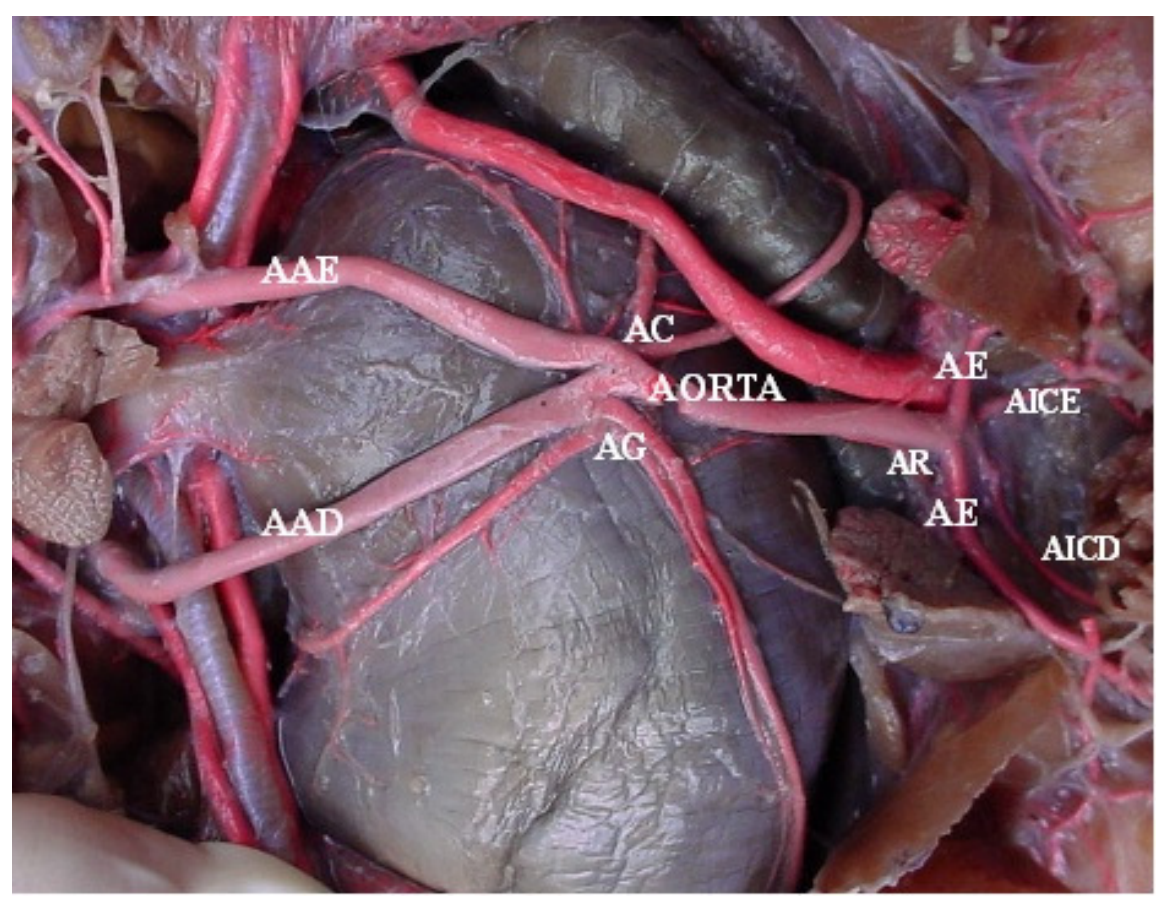

Figura 1: Fotografia de Podocnemis unifilis (AAD) arco aórtico direito, (AAE) arco aórtico esquerdo, (AORTA) artéria aorta, (AR) artéria renal, (AE) artéria epigástrica, (AICD) artéria ilíaca comum direita, (AICE) artéria ilíaca comum esquerda, (AC) artéria celíaca, (AG) artéria gástrica. 


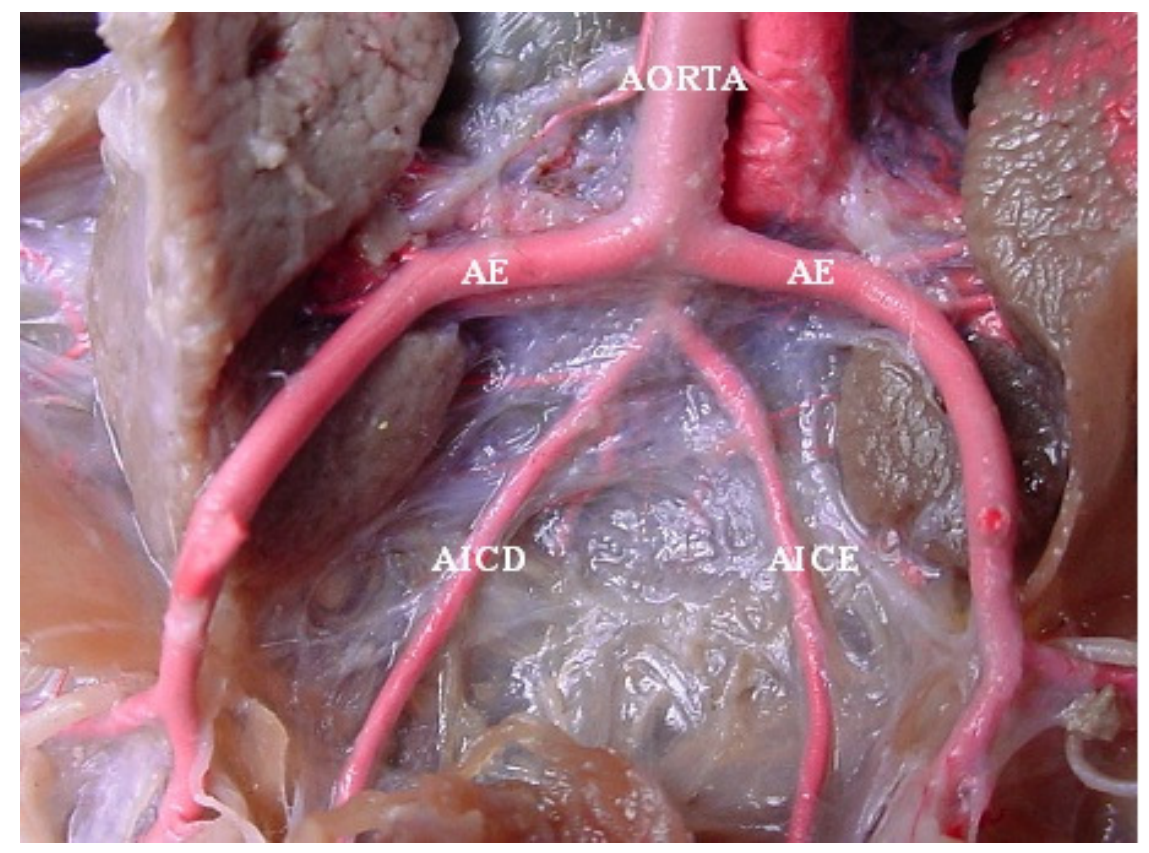

Figura 2: Fotografia de Podocnemis unifilis (AORTA) artéria aorta e ramificações, (AE) artéria epigástrica, (AICD) artéria ilíaca comum direita, (AICE) artéria ilíaca comum esquerda.

\section{DISCUSSÃO}

A origem e trajeto dos arcos aórticos direito e esquerdo afirmam que estes arcos após deixarem o coração possuem um trajeto dorso-cranial e posteriormente ventro-caudal próximo a parte cranial do estômago, tendo um ponto de união entre os arcos aórticos direito e esquerdo, próximo a parte caudal do estômago (NEAL e RAND, 1954 e OLIVEIRA et al., 2009)

Santos et al. (2003) também estudaram a anatomia vascular em Podocnemis expansa, mas o enfoque foi descrever as artérias coronárias, encontrando uma única artéria originada da face lateral direita do coração, na porção inicial do tronco braquiocefálico. 
SANTOS, A.L.Q. et al. Anatomia vascular de Podocnemis unifilis (Testudines - Pelomedusidae). Comportamento da artéria aorta. PUBVET, Londrina, V. 5, N. 16, Ed. 163, Art. 1103, 2011.

Em Podocnemis expansa, a artéria mesentérica cranial é responsável pela nutrição do mesentério, intestino delgado e parte do intestino grosso. No entanto, os autores relatam que a artéria mesentérica caudal é uma continuação da artéria celíaca (MORAES e SANTOS, 2004). A artéria mesentérica caudal originando-se, na maioria dos casos, da artéria ilíaca interna do antímero direito (RODRIGUES, MIGLINO e MELO, 2003). Em Trachemys scripta elegans a artéria mesentérica cranial executa o maior aporte da irrigação sanguínea do baço. No entanto, em $P$. geofroanus a artéria mesentérica caudal origina-se da artéria aorta (FARIA e MELO, 2007).

No arco aórtico esquerdo temos que Neal e Rand (1954), Faria e Mariana (2001) e este trabalho relatam que o arco aórtico esquerdo origina as artérias celíaca, porem o primeiro comenta que a mesentérica anterior também se origina do arco aórtico esquerdo, já Faria e Mariana (2001) e esta pesquisa informam que a mesentérica caudal e cranial apresenta-se como um segmento a partir da celíaca. No entanto Faria e Mariana (2001) usam artéria aorta esquerda ao invés de arco aórtico esquerdo e artérias mesentéricas inferior e superior não utilizando mesentéricas caudal e cranial.

Breland (1953) também afirma que a artéria celíaca origina-se do arco aórtico porém este arco é denominado de arco sistêmico, não dando ênfase no arco aórtico esquerdo.

Em Podocnemis expansa encontrou-se a artéria mesentérica cranial como um ramo do tronco celíaco-mesentérico e originando as artérias pancreaticoduodenal caudal que irrigam o duodeno; e a artéria mesentérica caudal origina-se da aorta ou da artéria ilíaca comum (SANTOS et al., 2004). Para Faria e Mariana (2001) em Geochelone carbonaria a artéria aorta esquerda ao invés de arco aórtico esquerdo e artérias mesentéricas inferior e superior não utilizando mesentéricas caudal e cranial.

Faria e Mariana (2001) encontraram a artéria pancreaticoduodenal caudal como sendo uma continuação da artéria celíaca, responsável pelo abastecimento sanguíneo do intestino grosso. Rodrigues, Miglino e Melo (2003) estudaram a vascularização artéria do trato gastrointestinal da Trachemys 
SANTOS, A.L.Q. et al. Anatomia vascular de Podocnemis unifilis (Testudines - Pelomedusidae). Comportamento da artéria aorta. PUBVET, Londrina, V. 5, N. 16, Ed. 163, Art. 1103, 2011.

scripta elegans e verificaram que a artéria pancreaticoduodenal caudal é originada da artéria hepática comum. Já em Phrynops geofroanus avaliou-se que a artéria pacreaticoduodenal caudal é originada da artéria mesentérica cranial.

Contrariando os resultados encontrados Harrison (1964) descreve que é a partir da artéria aorta dorsal que se tem a artéria celíaca. O autor comenta também que á artéria celíaca origina o ramo gástrico anterior, o ramo pancreáticoduodenal e o ramo hepático para os respectivos órgãos, e a artéria mesentérica anterior par o fígado e intestino delgado. Já esta pesquisa relata que é o arco aórtico esquerdo o responsável pelo surgimento da artéria gástrica, sendo as artérias pancreáticoduodenais cranial e caudal (irrigam baço, estômago e pâncreas, duodeno respectivamente) originárias das artérias celíaca.

Pereira et al. (2008) descrevem que a partir da união dos arcos aórticos direito e esquerdo tem-se a artéria aorta, entretanto, Baroudi (1965) relata a presença de duas aortas antes da união das mesmas, e após esta união temse a artéria torácica única que emite troncos e irriga as estruturas do organismo.

Os achados desta pesquisa corroboram com Hirano et al. (2009) que em Podocmenis unifilis observaram que as artérias renais se originam da face ventral da artéria aorta dorsal, e foram encontrados dois pares de artérias renais para cada rim, em um único exemplar, elas originaram uma artéria renal direita e duas esquerdas. A artéria gonadal surgiu a partir da artéria renal, e apenas um par penetrou pela face dorsal de cada gônada.

Para Storer et al. (1995) existe dois arcos aórticos que se unem e forma á aorta dorsal, contrariando em parte a pesquisa mostrada, pois esta denomina apenas de artéria aorta.

O trabalho presente descreve que a partir da união dos arcos aórticos direito e esquerdo tem-se a artéria aorta, entretanto Baroudi (1965) relata a presença de duas aortas antes da união das mesmas, e após esta união tem- 
SANTOS, A.L.Q. et al. Anatomia vascular de Podocnemis unifilis (Testudines - Pelomedusidae). Comportamento da artéria aorta. PUBVET, Londrina, V. 5, N. 16, Ed. 163, Art. 1103, 2011.

se a artéria torácica única que emitem troncos e irriga as estruturas do organismo.

\section{CONCLUSÕES}

Pode-se concluir que: a origem dos arcos aórticos obedece ao padrão reptiliano, cujo arco aórtico direito deixa o coração pelo lado esquerdo e o arco aórtico esquerdo pelo lado direito do ventrículo; o arco aórtico esquerdo origina as artérias gástricas, celíaca, já o arco aórtico direito não emite nenhum ramo antes da união com o arco aórtico esquerdo; a artéria aorta emite as artérias renais, epigástricas, caudal e ilíacas.

\section{REFERÊNCIAS}

AMABIS, J. M.; MARTHO, G. R. Curso básico de biologia - seres vivos. São Paulo: Moderna, 1990. v.2. 14-16/388 p.

ASHLEY, L. M. Laboratory anatomy of the turtle. Dubuque, Iowa: WM. C. Brown, 1969. 2634.p.

BAROUDI, R. Elementos de zoologia de cyclostomata a mammalia. 4ed. São Paulo: Nobel, 1965. v. 3.67. p.

BENZZONI, P. Compêndio de zoologia. Belo Horizonte: Livraria Cultural Brasileira, 1972. 175. p.

BRELAND, O. P. Manual of comparative anatomy. 2ed. Nova York: McGraw-Hill Book, 1953. 124. $\mathrm{p}$.

BURTON, M.; BURTON, R. Encyclopedia of reptiles, anphibians and other cold-blooded animals. San Sebastian: Tonsa, 1984. 121p.

CARR, A. Biblioteca da natureza. Rio de Janeiro: Livraria José Olympio, 1981. 151p.

FARIA, T. N.; MARIANA, A. N. B., Origem e ramificações das artérias aortas esquerda e dorsal do jabuti (Geochelone carbonaria, Spix, 1824). Brazilian Journal of Veterinary Research and Animal Science. São Paulo, v.38, n. 4, p.155-159, Jan. 2001.

FARIA, M. D. ; MELO, A. P. F. Topografia, morfologia e irrigação do Baço em Trachemys scripta elegans (WIED, 1838). Brazilian Journal Veterinary Research Animal and Sciense, São Paulo, v. 44, n.3, p. 167-173, Out. 2007.

FUENTE, F. R. A fauna- vida e costume dos animais selvagens. Rio de Janeiro: Salvat, 1982. v.4. 57p. 
SANTOS, A.L.Q. et al. Anatomia vascular de Podocnemis unifilis (Testudines - Pelomedusidae). Comportamento da artéria aorta. PUBVET, Londrina, V. 5, N. 16, Ed. 163, Art. 1103, 2011.

GRASSÉ, P. P. Zoologia - vertebrados reproducción, biologia, evolucion y sistemática agnatos, peces, anfibios y reptiles. Barcelona: Toray-masson, 1978. v3. 1-9.p.

HARRISON, B. M. Manual of comparative anatomy a general laboratory guide. 2ed. St. Louis: C.V. Mosby, 1964. 156p.

HICKMAN, C. P. Princípios de zoologia. (S.I): Ariel, s.d. 501-502 p.

HIRANO, L. Q. L.; SANTOS, A. L. Q.; PEREIRA, P. C.; SILVA, J. M. M.; KAMINISHI, Á. P. S.; FERREIRA, C. G. Anatomia vascular das artérias renais e gonadais de Podocnemis unifilis Schweigger, 1812 (Testudines, Pelomedusidae) Acta Scientiarum. Biological Sciences, Maringá, v.31, n.2, p.191-194, Fev. 2009.

HYMAN, L. H. Comparative vertebrate anatomy. Chicago: The University of Chicago press, 1957. 347-348p.

MORAES, F. M.; SANTOS, A. L. Q. Anatomia vascular da Podocnemis expansa (TestudinataPelomedusidae). Comportamento da Artéria Aorta. Horizonte Científico. Uberlândia, n. 3, p. 1-13, Jan. 2004.

MORANDINI. C. Zoologia. 2ed. São Paulo: Nobel S. A, 1968. v4. 1196p.

NEAL, H. V.; RAND, H. W. Comparative anatomy. New York: Blakiston Company, 1954. 356p.

NIELSEN, K. S. Animal physiology: adaptation and environmente. 3ed. New York: Cambridge University, 1986. 619p.

OLIVEIRA, S. C. R.; MACHADO JÚNIOR, A. A. N.; CARVALHO, R. C.; PEREIRA, L. A.; OliVEIRA, A. S.; SOUSA, A. L. Distribuição arterial dos principais vasos da cavidade celomática em Kinosternon sorpioides scorpioides. Ciência Animal Brasileira, Goiânia, v.10, n.3, p.893898, Jul./Set. 2009.

ORR, R. T. Biologia dos vertebrados. 5ed. São Paulo: Roca, 1986. 130p.

PEREIRA, H. C. SILVA JUNIOR, L. M. MUNDIM, A. V.; SANTOS, A. L. Q. Anatomia vascular de Phrynops geoffroanus Schweigger, 1812 (Testudines-Chelidae)- origem e ramificações das artérias mesentérica cranial e mesentérica caudal In: VIII Encontro Interno e XII Seminário de Iniciação Científica, 0438, 2008, Uberlândia. Anais... Universidade Federal de Uberlândia, 2008 , p. 1.

POUGH, F. H.; HEISER, J. B.; MCFARLAND, W. N. A vida dos vertebrados. São Paulo: Atheneu, 1993. 409p.

PRITCHARD, P. C. H. Encyclopedia of turtles. New Jersey: T. F. H., 1973. 326p.

RENNER, M., CUNHA, A. X. Guia de trabalhos práticos de zoologia. 15. ed. Coimbra: Atlântica, 1969. 341-415p.

RODRIGUES, R. F.; MIGLINO, M. A.; MELO, A. P. F. Vasculariazação arterial do trato gastrointestinal da Trachemys scripta elegans, Wied, 1838. Brasilian journal of veterinary research and animal science. São Paulo, n.40, p. 63-88, Out. 2003.

ROMER, A. S. Anatomia comparada (vertebrados). 4ed. México: Interamericana, 1973. 289-320p. 
SANTOS, A.L.Q. et al. Anatomia vascular de Podocnemis unifilis (Testudines - Pelomedusidae). Comportamento da artéria aorta. PUBVET, Londrina, V. 5, N. 16, Ed. 163, Art. 1103, 2011.

ROMER, A. S.; PARSONS, T. S. Anatomia comparada dos vertebrados. São Paulo: Atheneu, 1985. 381p.

SANTOS, A. L. Q.; ALVARENGA, G. J. R.; MORAES, F. M.; AVILA JUNIOR, R. H.; CARVALHO, S. F. M.; MAGALHÃES, L. M.; ANDRADE, M. B.; MARQUES, F. K.; DENADAI, J. Morfologia externa, topografia do coração e comportamento da artéria coronária de Podocnemis expansa (SCHWEIGGER, 1812). Bioscience Journal. Uberlândia, v. 19, n. 3, p. 103-108, Sept./Dec. 2003.

SANTOS, A. L. Q.; MUNDIM, A. V.; MORAES, F. M.; ALVARENGA, G. J. R.; MARQUES, F. K.; BORGES, K. M.; ALVES JÚNIOR, J. R. F. Origem e ramificações das artérias mesentéricas cranial e caudal em tartaruga da amazônia Podocnemis expansa - Schweigger, 1812, (Testudinata-Pelomedusidae). Archives of Veterinary Science, Curitiba, v.9, n.2, p.49-53, Out. 2004.

STORER, T. I.; USINGER, R. L., STEBBINS, R. C.; NYBAKKEN, J. W. Zoologia geral. 6ed. São Paulo: Nacional, 1995. 667p.

ZISWILLER, V. Zoologia especial dos vertebrados. Barcelona: Omega, 1978. v.1. 1-3p. 